\title{
Assessment of testis histopathological changes and spermatogenesis in male mice exposed to chronic scrotal heat stress
}

\author{
Tung Nguyen Thanh (D. Phuoc Dang Van • Thuan Dang Cong ID. Tam Le Minh ID. Quoc Huy Nguyen Vu (iD
}

T Nguyen Thanh (Corresponding author)

Institute of Biomedical Research, Hue University of Medicine and Pharmacy, Hue University, 6 Ngo Quyen Street, Hue, Vietnam.

Department of Histology, Embryology, Pathology and Forensic, Hue University of Medicine and Pharmacy, Hue University, 6 Ngo Quyen Street, Hue, Vietnam.

email: nttung@huemed-univ.edu.vn; nguyenthanhtung@hueuni.edu.vn

\section{T Dang Cong}

Department of Histology, Embryology, Pathology and Forensic, Hue University of Medicine and Pharmacy, Hue University, 6 Ngo Quyen Street, Hue, Vietnam.

\section{P Dang Van}

Institute of Biomedical Research, Hue University of Medicine and Pharmacy, Hue University, 6 Ngo Quyen Street, Hue, Vietnam.

Forensic Medicine Center, Thua Thien Hue Province, Vietnam.

\section{T Le Minh - QH Nguyen Vu}

Department of Obstetrics and Gynecology, Hue University of Medicine and Pharmacy, Hue University, 6 Ngo Quyen Street, Hue, Vietnam.

Received: April 04, 2020 - Accepted: April 20, 2020 - Published Online: April 22, 2020

\begin{abstract}
Elevation of scrotal temperature may be injurious to spermatogenesis and leading cause male infertility. Scrotal heat stress reduces the number and motility of spermatozoa, fertilization ability of the surviving sperm and poor fertilization-embryo. This study was designed to investigate the effect of heat stress (at $37{ }^{\circ} \mathrm{C}, 40{ }^{\circ} \mathrm{C}$ and $43{ }^{\circ} \mathrm{C}$ ) on histopathological features of testicular tissue in scrotal heat exposed male mice. Experimental and control groups were sacrificed after completion of five weeks heat exposure period. The testes were fixed and stained hematoxylin-eosin (H\&E) for qualitative and quantitative analysis of histopathological alterations and spermatogenesis according to Johnson scoring system. The results indicated that mice exposed to heat-stress mice exhibited degenerated and disorganized features of spermatogenic epithelium and reduced spermatogenic cells. Heat stress exposure $\left(40{ }^{\circ} \mathrm{C}\right.$ and $43{ }^{\circ} \mathrm{C}$ ) shows the significantly reduced Johnson score compared to the control condition $(P<0.05$ and $P<0.001$, respectively). Meanwhile, scrotal heat exposure at $37{ }^{\circ} \mathrm{C}$ did not reach significantly changes in Johnsen's testicular histopathological score. The seminiferous tubule structure and spermatogenesis were completely disrupted in mice exposed to $43{ }^{\circ} \mathrm{C}$. Percentage of high Johnsen score point was decreased in mice in heat-stress exposure group, while the ratio of low Johnsen score points was gradually increase. Spermatogenesis in male mice exposed to chronic scrotal heat stress is disrupted at $43{ }^{\circ} \mathrm{C}$. In conclusion, this study attempted to develop an animal model for studying the male reproductive
\end{abstract}

system. Johnsen scores system was standardized to assess murine testicular histopathology in the seminiferous tubule cross-section. Collectively, these results indicated a negative impact on histopathological alterations and spermatogenesis arrest following chronic scrotal heat stress.

Keywords: Johnson scoring system, male reproductive system, mouse model, testicular tissue

\section{Introduction}

The scrotal temperature in most mammals is normally below the core body temperature by $2-8{ }^{\circ} \mathrm{C}$, playing an important role in spermatogenesis that occurs in the testes normally. Increases in testicular temperature may be detrimental to spermatogenesis and ultimately cause problems in fertility (Shadmehr et al 2018; Lin et al 2015). Elevation of scrotal temperature interrupts spermatogenesis, resulting in reduced the number and motility of spermatozoa, fertilization ability of the surviving sperm and poor fertilization-embryo (Shadmehr et al 2018; Rasooli et al 2010). High scrotum temperature increases oxidation, resulting in disrupted thermoregulation of the testicle such as reduction of antioxidant enzymes production and heat shock protein production, and an increase in apoptosis and cell death (Kumar Roy et al 2016). Heat stress reduces the ability to regulate the temperature of the scrotum, causes oxidative stress, thus causing cellular reactions including mitochondrial dysfunction, increase the ROS and reduces the production of 
anti-ROS enzymes, reduces the increased apoptotic rate. Besides, heat stress acts inhibitory on the structure and function of the testes, leading to decrease male fertility (Rasooli et al 2010).

Scrotal heat stress causes a variety of mechanisms that occur in the testes including oxidative stress response, heat shock response, cell cycle checkpoints, DNA repair and apoptosis and cell death (Lin et al 2015; Zhang et al 2015). Scrotal heat stress interrupts spermatogenesis in male mice (Lin et al 2015). Spermatogonial germ cells are also influenced by heat stress, resulting in the elimination and absence of them in the seminiferous tubules, Sertoli and Leydig cells are degenerated (Rasooli et al 2010).

Mice are animal models commonly used in the study of the effects of heat stress because they are readily available, easy to find, cost-effective, easy to handle and have similarities in thermostats such as a human. Several studies have used experimental mouse models to assess environmental stress and exercise stress (Rakesh et al 2014). Previous research has evaluated the testicular response after transient scrotal temperature exposure leading to germ cell death and complex stress response (Paul et al 2009). However, animal model for heat stress study is not yet well established. This study aims to establish a mouse model for scrotal heat stress and investigate the histomorphometric change of testicular tissue in scrotal heat-stress exposed male mice based on Johnsen score of testicular histopathology.

\section{Materials and Methods}

\section{Animals}

Adult male Swiss mice with 8-10 weeks old (20-23g) were purchased from Pasteur Institute of Nha Trang, Viet Nam. Mice were kept in animal facility in a controlled temperature $\left(25 \pm 1{ }^{\circ} \mathrm{C}\right)$ and photoperiod (12h light: $12 \mathrm{~h}$ dark), with free access to food and water. Animals were randomly divided into 4 groups of 5 mice each: Control group, $37{ }^{\circ} \mathrm{C}$ heat exposure group, $40{ }^{\circ} \mathrm{C}$ heat exposure group, $43{ }^{\circ} \mathrm{C}$ heat exposure group.

\section{Experimental heat stress}

The lower half of the mouse's body, including the scrotum, was submerged in a temperature-controlled water bath $\left(37^{\circ} \mathrm{C}, 40^{\circ} \mathrm{C}\right.$ or $\left.43{ }^{\circ} \mathrm{C}\right)$ for 10 minutes, twice per day, at 10 minutes intervals, 6 days per week for 5 weeks. Control mice were treated within the same way, but in water bath maintained at $25{ }^{\circ} \mathrm{C}$. After having the bath, mice were dried and examined for any injury or redness to the scrotal skin before returned to their cages. The four groups were kept in different cages and had free access to water and food. All animals were cared for under the identical environmental conditions. The mice were monitored their general health.
After completing the heat exposure experiment after 5 weeks, all mice were sacrificed under anesthesia. Their testis tissue was harvested for histological morphometric analysis.

\section{Tissue processing and hematoxylin-eosin staining}

At the end of the surgical procedure, the testicular specimens were individually immersed into $4 \%$ buffered formaldehyde, dehydrated with graded concentrations of ethyl alcohols and embedded tissues into paraffin blocks. Paraffin sections of $5 \mu \mathrm{m}$ thick were obtained transferred onto gelatincoated slides, deparaffinized with xylene and then rehydrated through a descending series of ethanol and water. Slides were stained with hematoxylin and eosin (H\&E) and observation under a light microscope for histopathological analysis.

\section{Histological morphometric analysis}

The testicular tissue was evaluated in random order by a pathologist, who was blinded to the study group allocations with Zeiss Axioplan-2 Imaging light microscopy (KS-300 Imaging System). Testicular damage and spermatogenesis were assessed histopathologically using Johnsen's mean testicular biopsy score (MTBS) (Johnsen 1970). By using a 40x magnification, thity tubules for each animal were graded and each tubule was given a score from 1 to 10 based on the presence or absence of germ cell types in the testicular seminiferous tubules such as spermatozoa, spermatids, spermatocyte, spermatogonia, germ cells and Sertoli cells to evaluate histology. A higher Johnsen's score indicates a better status of spermatogenesis, while a lower score refers to more severe dysfunction. Score 1 means no epithelial maturation considered for the tubules with complete inactivity while a score 10 means full epithelial maturation considered for the tubules with maximum activity (Table 1 ).

\section{Statistical analysis}

Results were analyzed using SPSS version 18. All data were reported as mean \pm standard deviation (SD). To evaluation of significant differences, the comparison of means between every two experimental groups was done by a computer program for Student's t-test. Differences were considered to be statistically significant if $P<0.05$.

\section{Results}

\section{Heat stress-induced spermatogenesis complications}

Mice without heat stress (control group: bathing in 25 ${ }^{\circ} \mathrm{C}$ water) showed the complete process of spermatogenesis in which progenitor spermatogonia develop into mature spermatozoa in the seminiferous tubules (Figure 1a). The germinal epithelium including of cells at different stages of spermatogenic development (spermatogonia, primary spermatocytes, secondary spermatocytes, spermatids, and 
spermatozoa) located in invaginations or in dilations between of Sertoli cells (Figure 1b). Meanwhile, the heat-stress exposed mice (all experimented groups) exhibited degenerated and disorganized features of spermatogenic epithelium and reduced spermatogenic cell numbers (Figure $1 \mathrm{c}$ and $1 \mathrm{~d})$.

Table 1 Histological classification of seminiferous tubular cross-sections according to the Johnsen scoring system.

\begin{tabular}{ll}
\hline Score & \multicolumn{1}{c}{ Description } \\
\hline 10 & $\begin{array}{l}\text { Complete spermatogenesis with many spermatozoa. Germinal epithelium organized in a } \\
\text { regular thickness leaving an open lumen. } \\
\text { Many spermatozoa present but germinal epithelium disorganized with marked sloughing or } \\
9\end{array}$ \\
& obliteration of lumen. \\
7 & Only a few spermatozoa present in the section. \\
6 & No spermatozoa and only a few spermatids present. \\
5 & No spermatozoa, no spermatids but several or many spermatocytes present. \\
4 & Only few spermatocytes and no spermatids or spermatozoa present. \\
3 & Spermatogonia are the only germ cells present. \\
2 & No germ cells but Sertoli cells present. \\
1 & No cells in the tubular section \\
\hline
\end{tabular}
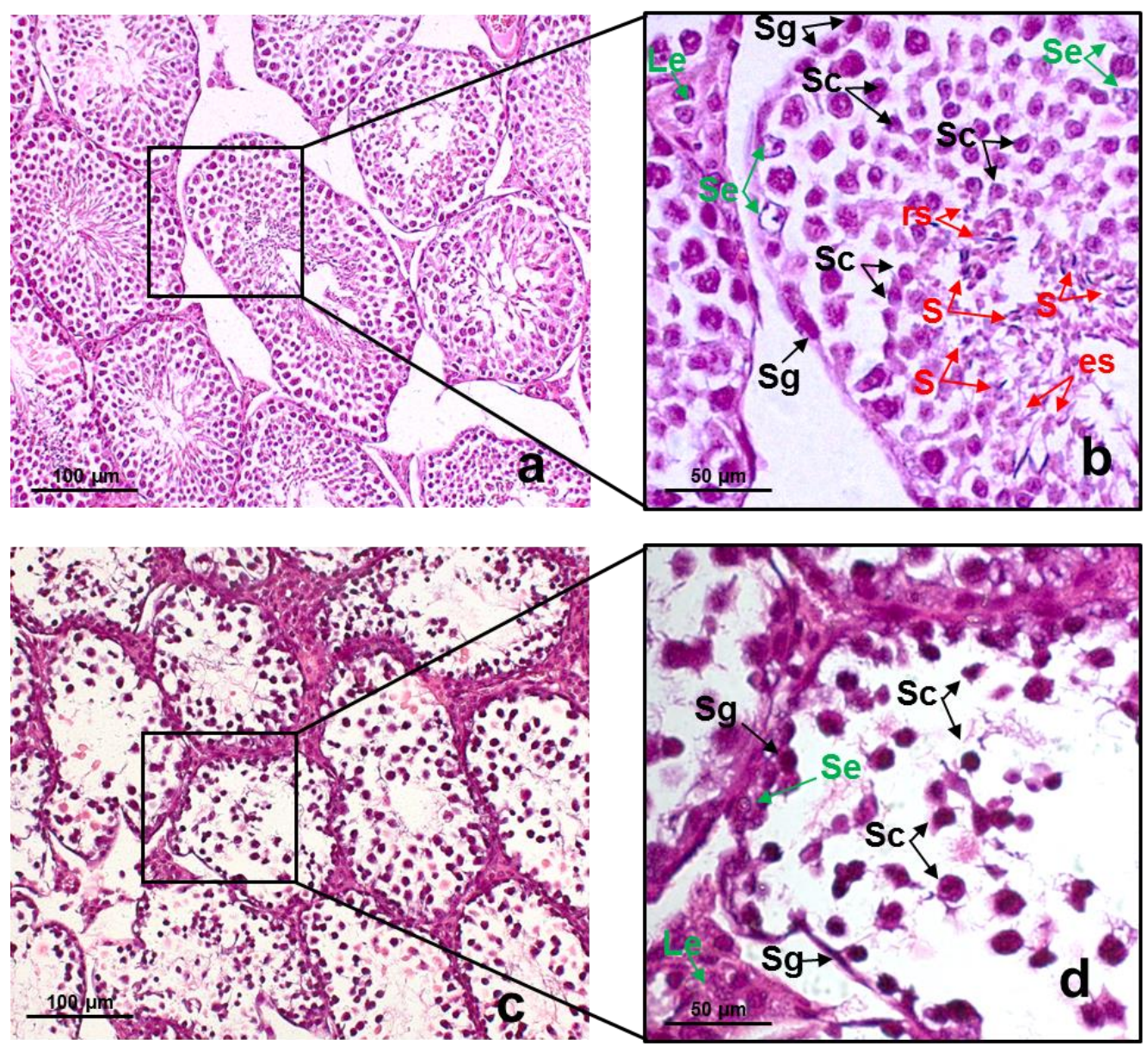

Figure 1 Testicular structure and spermatogenesis in controlled and heat-stress exposed male mice. a. Testicular structure in controlled male mice b. Complete spermatogenesis occurs in the seminiferous tubules c. Testicular structure in heat-stress exposed male mice d. Spermatogenesis arrest occurs in the seminiferous tubules from heat-stress exposed mice; Le: Leydig cells, Se: Sertoli cells, Sg: Spermatogonia, Sc: Spermatocytes, rs: round spermatids, es: elongating spermatids, S: Spermatozoa. 
Standardization of Johnsen scores in the seminiferous tubule cross-sections

Histological structure and spermatogenesis in mice were evaluated by the Johnsen scoring system. The seminiferous tubules were graded (1 to 10) according to reduction in the number and density of germ cells from the lumen of seminiferous tubules. Standardization of Johnsen scores in the seminiferous tubule cross-sections of control and heat exposed group mice was shown in Figure 2. Johnsen scores 8,9 , and 10 indicate spermatogenesis with a few to many spermatozoa present in a section of seminiferous tubular
(Figure 2a, 2b and 2c). Scores 6 and 7 indicate seminiferous tubular with no spermatozoa, but spermatids present (Figure $2 \mathrm{~d}$ and 2e). Seminiferous tubular with no spermatozoa, no spermatids, but spermatocyte present is evaluated as scores 4 and 5 (Figure $2 \mathrm{~g}$ and 2f). Seminiferous tubular with only spermatogonia as germ cell presence is evaluated as score 3 (Figure 2h), meanwhile tubular with no germ cells but Sertoli cells present is given a score 2 (Figure 2i). Seminiferous tubular with a complete absence of cells in the seminiferous tubules is evaluated as score 1 (Figure 2j).
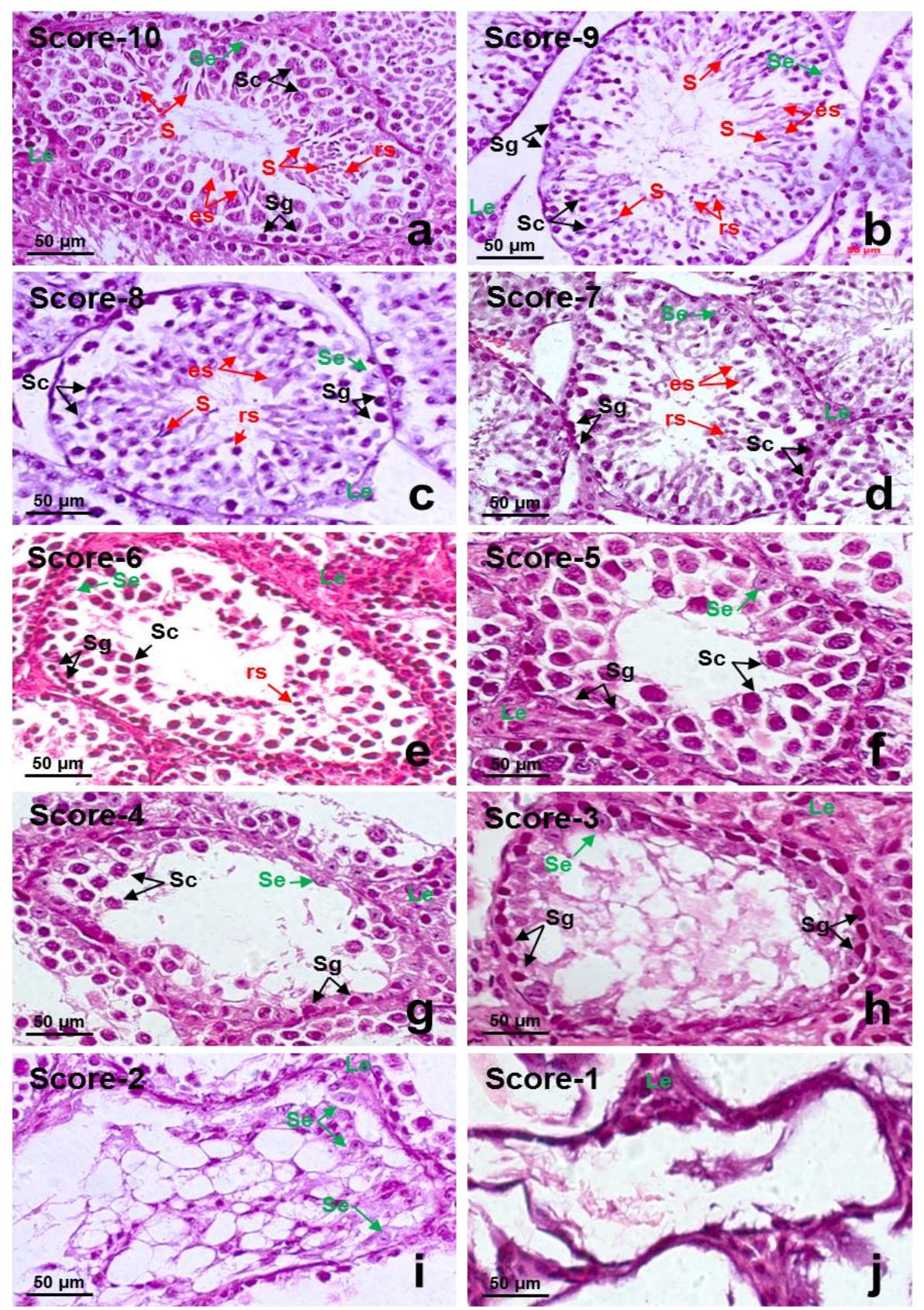

Figure 2 Standardization of Johnsen scores in the seminiferous tubule cross-sections of control and heat exposed group mice stained with hematoxylin-eosin (HE). a. Johnsen score 10; b. Johnsen score 9; c. Johnsen score 8; d. Johnsen score 7; e. Johnsen score 6; f. Johnsen score 5; g. Johnsen score 4; h. Johnsen score 3; i. Johnsen score 2; j. Johnsen score 1. Le: Leydig cells, Se: Sertoli cells, Sg: Spermatogonia, Sc: Spermatocytes, rs: round spermatids, es: elongating spermatids, S: Spermatozoa. 
Histological evaluation of seminiferous tubules in heat-stress exposed mice

Box and whisker plots in Figure 3 show the Johnsen score of seminiferous tubule cross-sections in normal and heat-stress exposed male mice. The result shows that there was a negative effect of high temperature on testicle structure and spermatogenesis in male mice. Johnsen score in the control group (exposed to $25^{\circ} \mathrm{C}$ water) was $8.46 \pm 1.05$, while the Johnsen score of the $37{ }^{\circ} \mathrm{C}$-heat exposed groups was lower $(7.38 \pm 1.13)$ but no statistically significant difference $(P>$ $0.05)$. Johnsen score in heat-exposed groups at $40{ }^{\circ} \mathrm{C}$ and 43 ${ }^{\circ} \mathrm{C}$ were $4.94 \pm 1.39$ and $2.46 \pm 1.20$, respectively. Heat stress exposure in $40{ }^{\circ} \mathrm{C}$ and $43{ }^{\circ} \mathrm{C}$ groups were both significantly reduced Johnson score compared to the control group $(P<$ 0.05 and $P<0.001$, respectively).
Frequency distribution of Johnsen scores in heat-stress exposed mice

The frequency distribution of Johnsen scores of seminiferous tubular cross-sections in controlled and heatstress exposed male mice was shown in Figure 4. This result revealed that scores 7-8 and 9-10 had the highest prevalence in the control group, with a relative frequency of $49.6 \%$ and $48.8 \%$ respectively, compared to $62.4 \%$ and $15.2 \%$, respectively, in the $37^{\circ} \mathrm{C}$ group. In the $40{ }^{\circ} \mathrm{C}$ group, scores 56 and 3-4 had the highest prevalence in the control group, with a relative frequency of $54.4 \%$ and $27.2 \%$ respectively. In the $43^{\circ} \mathrm{C}$ group, scores 1-2 and 3-4 had the highest prevalence in the control group, with a relative frequency of $54.4 \%$ and $42.2 \%$ respectively. Heat-stress exposure decreased the percentage of high Johnsen points, gradually increasing the ratio of low Johnsen points.

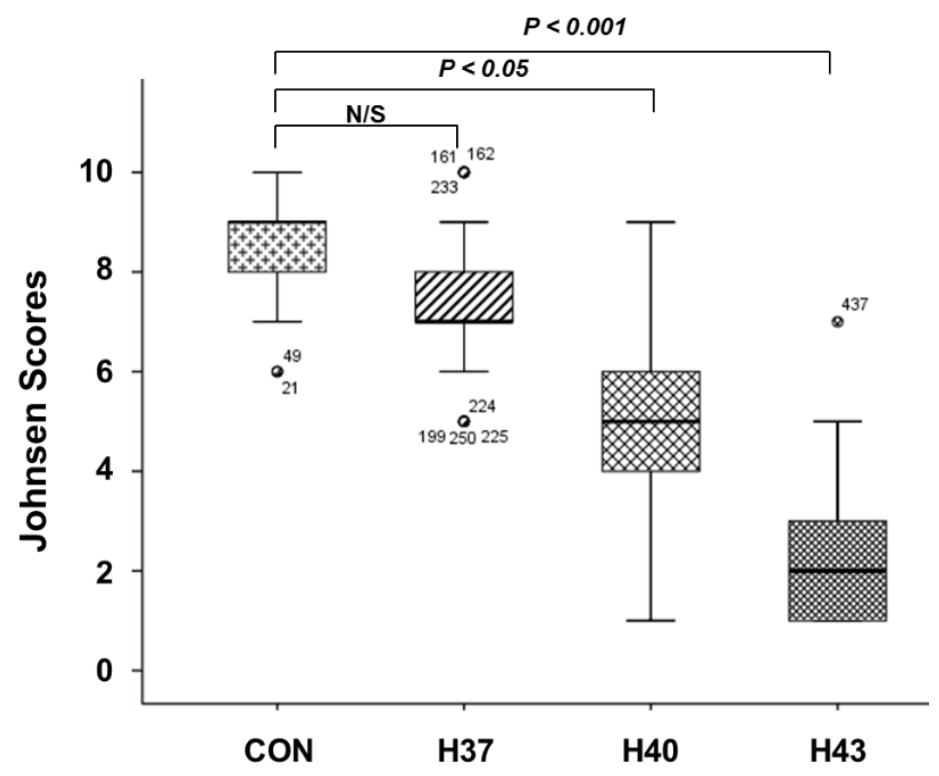

Figure 3 Box and whisker plots showing the Johnsen score of seminiferous tubular cross-sections in controlled and heat-stress exposed male mice. CON: Controlled group; H37, H40, H43: Heat-stress exposed groups at 37,40 and $43{ }^{\circ} \mathrm{C}$.

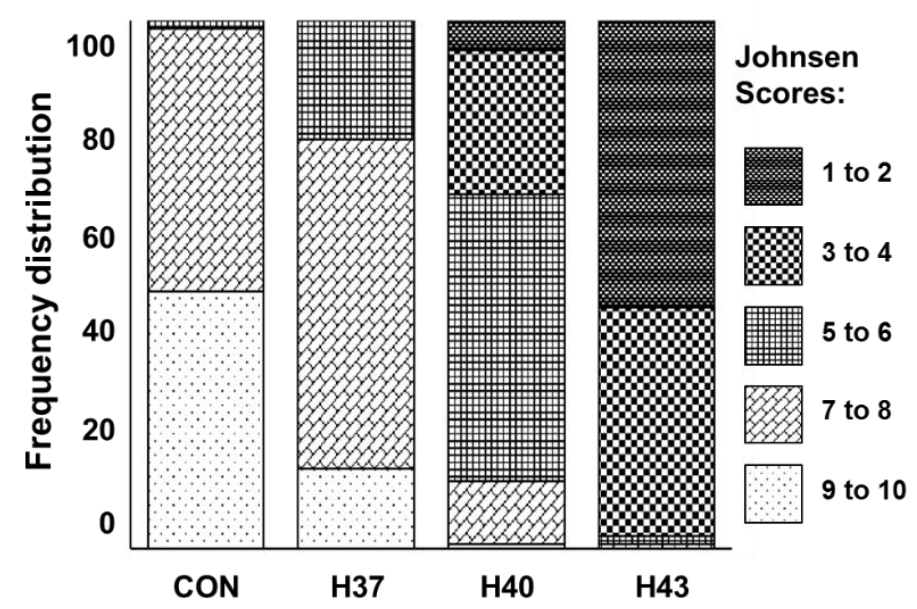

Figure 4 Histograms showing the frequency distribution of Johnsen scores of seminiferous tubular cross-sections in controlled and heat-stress exposed male mice. CON: Controlled group; H37, H40, H43: Heat-stress exposed groups at 37, 40 and $43{ }^{\circ} \mathrm{C}$. 
Spermatozoa cell number in tubular cross-section of heatstress exposed mice

The mean of spermatozoa number per tubular crosssection in controlled and heat-stress exposed male mice was shown in Figure 5. In the control group, there were 14.6 \pm 10.1 spermatozoa per tubular cross-section. Heat stress exposed at $37^{\circ} \mathrm{C}$ was significantly reduced the number of spermatozoa in tubular cross-section $(6.5 \pm 8.2)$ compared to those in a control group $(P<0.001)$. There were a few spermatozoa in tubular cross-section in the $40{ }^{\circ} \mathrm{C}$ group $(0.5 \pm 2.3)$, while no spermatozoa were observed in the seminiferous tubules in the $43{ }^{\circ} \mathrm{C}$ group.

\section{Discussion}

Mammalian spermatogenesis is a complex system within structurally well-designed seminiferous tubules. During the process of spermatogenesis, the diploid spermatogonia (Spermatogonial stem cells) differentiate into primary spermatocytes, then undergo the first meiotic division into two secondary spermatocytes. Each secondary spermatocytes go through the second meiosis division to produce haploid daughter spermatids. Spermatid transforms into spermatozoa by the process of metamorphosis (spermiogenesis) (Hunter et al 2012). Globally, animal models have commonly been used to address a variety of biomedical research including, from basic science to immunology and infectious disease, oncology, and behavior or therapies. Laboratory mice have highly homogeneous genetic composition and have been developed to be the powerhouse for biomedical research (Barre-Sinoussi and Montagutelli 2015). Animal model, especially mouse model was investigated for the study of human infertility or spermatogenesis, such as the genes involved in male infertility (Jamsai and O'Bryan 2011), effects of testes hyperthermia (Kumar Roy et al 2016), cisplatin-induced testicular injury (Whirledge et al 2015). In this study, the mouse model was established to study the effect of scrotal heat stress on spermatogenesis and male fertility.

Johnsen's score evaluation system was used in several studies to assess testicular histology, a relatively complete scoring system with a full level of testicular histological scores given a score of 10 to 1 with a decrease in the number of cells in the lumen of the spermatogenesis (Johnsen 1970). This quantitative histological grading system is a reliable, easily obtained and simple tool to prognosis for reproductive capacity in men (Dohle et al 2012; Teixeira et al 2019). Johnsen's score is also a histopathological predictor of semen quality after a number of treatment indications in male infertility patients to analyze and determine positive prognostic values that help improve fertility (Teixeira et al 2019). In this study, we applied the criteria formulated by Johnsen to assess murine testicular histopathology affected by scrotal heat stress. The result showed that Johnsen's score was significantly reduced in the heat stress groups compared with those in the control group, increasing the possibility of infertility in male mice.

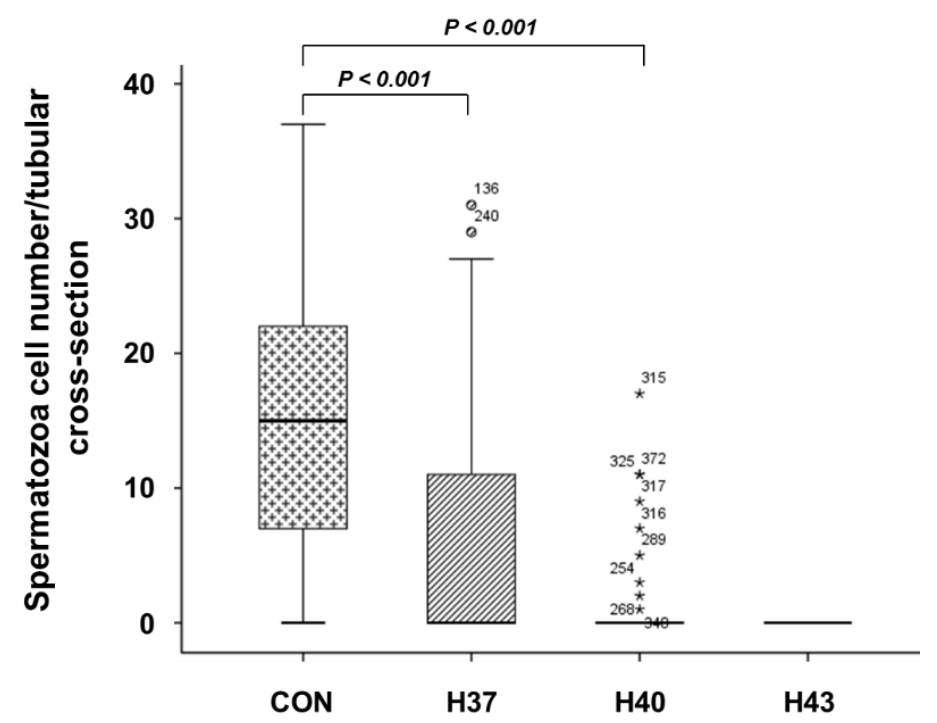

Figure 5 Box and whisker plots showing mean spermatozoa number per tubular cross-section in normal and heat-stress exposed male mice. CON: Controlled group; H37, H40, H43: Heat-stress exposed groups at 37,40 and $43^{\circ} \mathrm{C}$.

The heat stress has been known as a risk factor of male infertility. However, the exact mechanism causing impaired spermatogenesis is still unclear. Testicular histology after thermal exposure had changes such as decreased epithelial thickness, appearance of cellular debris, fragmented cells, and absence of sperm and spermatocytes (Setchell 2006; Gao et al 
2012). The impact of prolonged heat stress negatively affects sperm quality and quantity, testicular structure with disruption of the surrounding epithelium (Setchell 2006). In addition, scrotal heat stress reduces testicular weight, mitochondrial degeneration, dilatation of the smooth endoplasmic reticulum and Leydig cells lose the function of supporting stem cells (Durairajanayagam et al 2015). Findings from this study support that the testicular structure was disorganized germinal epithelium with marked sloughing or obliteration of lumen; spermatogenesis was interrupted with the absence of many types of sperm cells in male mice after chronic scrotal heat stress exposed for 5 weeks.

In conclusion, this study standardized the Johnsen score system to assess murine testicular histopathology in the seminiferous tubule cross-sections. There was evidence for negative effects on histopathological alterations and spermatogenesis arrest following chronic scrotal heat stress. In addition, this study attempted to develop an animal model for studying the male reproductive system.

\section{Acknowledgments}

This study was supported by the Hue university-level research projects in science and technology (DHH 2019-0488). The authors would like to thank Dr. Dang Thi Anh Thu, Department of Environmental \& Occupational Health, Faculty of Public Health, Hue University of Medicine and Pharmacy, for her revising this manuscript.

\section{Conflict of Interest}

The authors declare no conflict of interest.

\section{References}

Barre-Sinoussi F, Montagutelli X (2015) Animal models are essential to biological research: issues and perspectives. Future Sci OA 1:FSO63.

Dohle GR, Elzanaty S, van Casteren NJ (2012) Testicular biopsy: clinical practice and interpretation. Asian J Androl 14:88-93.

Durairajanayagam D, Agarwal A, Ong C (2015) Causes, effects and molecular mechanisms of testicular heat stress. Reprod Biomed Online 30:14-27.
Gao J, Zuo Y, So KH, Yeung WS, Ng EH, Lee KF (2012) Electroacupuncture enhances spermatogenesis in rats after scrotal heat treatment. Spermatogenesis 2:53-62.

Hunter D, Anand-Ivell R, Danner S, Ivell R (2012) Models of in vitro spermatogenesis. Spermatogenesis 2:32-43.

Jamsai D, O'Bryan MK (2011) Mouse models in male fertility research. Asian J Androl 13:139-151.

Johnsen SG (1970) Testicular biopsy score count--a method for registration of spermatogenesis in human testes: normal values and results in 335 hypogonadal males. Hormones 1:2-25.

Kumar Roy V, Marak TR, Gurusubramanian G (2016) Alleviating effect of Mallotus roxburghianus in heat-induced testicular dysfunction in Wistar rats. Pharm Biol 54:905-918.

Lin C, Shin DG, Park SG, Chu SB, Gwon LW, Lee JG, Yon JM, Baek IJ, Nam SY (2015) Curcumin dose-dependently improves spermatogenic disorders induced by scrotal heat stress in mice. Food Funct 6:3770-3777.

Paul C, Teng S, Saunders PT (2009) A single, mild, transient scrotal heat stress causes hypoxia and oxidative stress in mouse testes, which induces germ cell death. Biol Reprod 80:913-919.

Rakesh V, Stallings JD, Reifman J (2014) A virtual rat for simulating environmental and exertional heat stress. J Appl Physiol 117:12781286.

Rasooli A, Taha Jalali M, Nouri M, Mohammadian B, Barati F (2010) Effects of chronic heat stress on testicular structures, serum testosterone and cortisol concentrations in developing lambs. Anim Reprod Sci 117:55-59.

Setchell BP (2006) The effects of heat on the testes of mammals. Animal Reproduction 3:81-91.

Shadmehr S, Fatemi Tabatabaei SR, Hosseinifar S, Tabandeh MR, Amiri A (2018) Attenuation of heat stress-induced spermatogenesis complications by betaine in mice. Theriogenology 106:117-126.

Teixeira TA, Pariz JR, Dutra RT, Saldiva PH, Costa E, Hallak J (2019) Cut-off values of the Johnsen score and Copenhagen index as histopathological prognostic factors for postoperative semen quality in selected infertile patients undergoing microsurgical correction of bilateral subclinical varicocele. Transl Androl Urol 8:346-355.

Whirledge SD, Garcia JM, Smith RG, Lamb DJ (2015) Ghrelin partially protects against cisplatin-induced male murine gonadal toxicity in a GHSR-1a-dependent manner. Biol Reprod 92:76.

Zhang MH, Shi ZD, Yu JC, Zhang YP, Wang LG, Qiu Y (2015) Scrotal heat stress causes sperm chromatin damage and cysteinyl aspartate-spicific proteinases 3 changes in fertile men. J Assist Reprod Genet 32:747-755. 